\title{
A MMSE-based Beamforming Algorithm for MIMO Point-to-Point Full-Duplex Communication Systems
}

\author{
Tu Bui-Thi-Minh", Xung Le ${ }^{\#}$, Vien Nguyen-Duy-Nhat ${ }^{\#}$ \\ \# Department of Electronics and Telecommunications Engineering, \\ University of Science and Technology - The University of Danang, VietNam \\ E-mail: btmtu@dut.udn.vn,lxung@dut.udn.vn,nhatvien@gmail.com
}

\begin{abstract}
In this paper, we focus on the precoding design for sum rate maximization while considering the effects of residual SI for point - to-point multiple input/multiple output (MIMO) Full-Duplex systems. The MMSE-based beamforming algorithm was proposed to cancel the SI. The results shown that, the self-interference cancellation is done by matrix precoding at the transmitter if the total number of transmitting antenna of two nodes is greater than the number of receiving antenna of one node. The Bit Error Rate (BER) was also evaluated in the simulation.
\end{abstract}

Keywords-MIMO, full-duplex, self-interference, beamforming, precoding, postcoding, MMSE.

\section{INTRODUCTION}

The capacity of system became the one of the most important features of next generation wireless communications networks because of the explosive demand for data. Key techniques in advanced wireless networks improved the capacity by increasing spectrum efficiency and reducing latency. The most common radio transmission technique is haft-duplex, in which the transmit and receive signals allocate two distinct frequency bands. The advantage of this method is that transmitting and receiving signals do not cause mutual interference, however, low spectrum utilization efficiency. In addition, another haft-duplex type has also been applied, which is the transmit and receive signals allocated in two different time slots. In this type, interference is avoided but it is not efficient in terms of time [1].

Full-duplex transmission is considered a double effective technique compared to haft-duplex. In this technique, the transmit and receive signals are transmitted in the same frequency at the same time. However, this technique exists self-interference (SI) signal from the transmitter antenna to the receiving antenna of the device itself. Therefore, fullduplex communication must conduct interference cancelation in various forms in different layers [2], [3].

Multi input / multiple output (MIMO) technology has been shown to have high spectral efficiency in wireless communication systems [4]. The works [5], [6] focus on two main problems in multi-antenna radio transmission systems, namely, capacity optimization and power control. Zero- forcing coding is one of the underlying techniques for solving this problem [7]. Block-diagonal is a pre-coding technique that forces the matrix's diagonal interference signals to zero to eliminate these components.

Authors in [8] - [11] considered the pre-coding technique for full-duplex transmission using a transistor focused on selfinterference signaling. Unlike these above works, this article focuses on point-to-point full-duplex communication, designs pre/post coding matrices to maximize system capacity.

The remaining parts of this paper are organized as follows. Section II delineates the system model of full-duplex MIMOOFDM. The proposed beamforming matrixes design is developed in Section III. Analytical and empirical results with relevant discussions are located in Section IV. Finally, Section V provides some concluding remarks of this research work.

Notations: $\mathbf{X} \in \mathbb{C}^{r \times c}$ denote the complex matrix with $r$ rows and $c$ columns, $\mathbf{X}^{H}$ is the transpose and conjugate transpose (Hermitian operator) of the matrix $\mathbf{X}$. $\|\mathbf{X}\|$ is the determinant of the matrix $\mathbf{X}$. $E($.$) stands for the expectation$ operator. $\operatorname{rank}(\mathbf{X})$ and $\operatorname{trace}(\mathbf{X})$ denote the rank and trace operator of the matrix $\mathbf{X}$, respectively. $\mathbf{I}_{\mathrm{y}}$ is the identity matrix having $y$ rows. And blkdiag $(\mathbf{X}, \mathbf{Y})$ is block diagonal operation from the matrixes $\mathbf{X}$ and $\mathbf{Y}$.

\section{SYSTEM MODEL}

In this paper, we consider a MIMO-OFDM full-duplex system as illustrated in Fig. 1, each node equips $n_{T}$ transmit antennas and $n_{R}$ receive antennas. 

by

The received signal at node 1 and node 2 can be expressed

$$
\begin{aligned}
& \mathbf{r}_{1}=\mathbf{H}_{1} \mathbf{V}_{1} \mathbf{x}_{1}+\mathbf{G}_{2} \mathbf{V}_{2} \mathbf{x}_{2}+\mathbf{z}_{1}, \\
& \mathbf{r}_{2}=\mathbf{H}_{2} \mathbf{V}_{2} \mathbf{x}_{2}+\mathbf{G}_{1} \mathbf{V}_{1} \mathbf{x}_{1}+\mathbf{z}_{2},
\end{aligned}
$$

where $\mathbf{x}_{1}, \mathbf{x}_{2} \in \mathbb{C}^{n_{T} \times 1}$ are the transmitted signal vectors from node 1 and node 2 , respectively; $\mathbf{V}_{1}, \mathbf{V}_{2} \in \mathbb{C}^{n_{T} \times n_{T}}$ denote the precoding matrices at node 1 and node 2, respectively; $\mathbf{G}_{1}, \mathbf{G}_{2} \in \mathbb{C}^{n_{R} \times n_{T}}$ stand for the matrices of channel gain from node 1 to node 2 and vice versa; $\mathbf{H}_{1}, \mathbf{H}_{2} \in \mathbb{C}^{n_{R} \times n_{T}}$ are the selfinterference matrices of channel gain at node 1 and node 2 , respectively; $\mathbf{z}_{1}, \mathbf{z}_{2} \in \mathbb{C}^{n_{R} \times 1}$ are additive white Gaussian noise (AWGN) with variance $\sigma_{1}^{2} \mathbf{I}_{R}, \sigma_{2}^{2} \mathbf{I}_{R}$ at node 1 and node 2 , respectively.

In (1) and (2), the first component on the right side is the self-interfering signal, the second component on the right side is the desired signal. Pre/post-coding techniques are applied to eliminate this first component in full-duplex communication system.

The desired signal can be restored by multiplying the post coding matrix by the following:

$$
\begin{aligned}
& \mathbf{y}_{1}=\mathbf{T}_{1} \mathbf{r}_{1}=\mathbf{T}_{1}\left(\mathbf{H}_{1} \mathbf{V}_{1} \mathbf{x}_{1}+\mathbf{G}_{2} \mathbf{V}_{2} \mathbf{x}_{2}+\mathbf{z}_{1}\right), \\
& \mathbf{y}_{2}=\mathbf{T}_{2} \mathbf{r}_{2}=\mathbf{T}_{2}\left(\mathbf{H}_{2} \mathbf{V}_{2} \mathbf{x}_{2}+\mathbf{G}_{1} \mathbf{V}_{1} \mathbf{x}_{1}+\mathbf{z}_{2}\right) .
\end{aligned}
$$

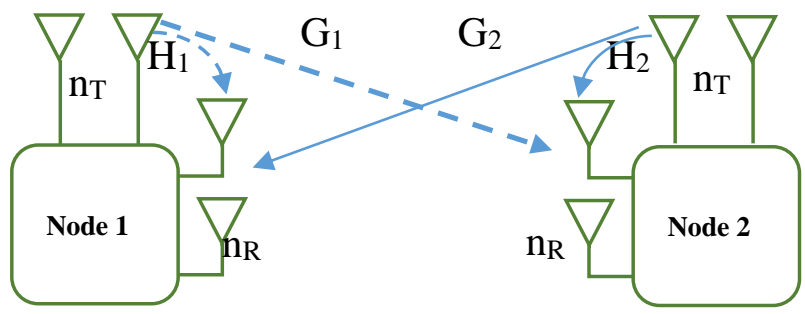

Fig. 1 System model of full-duplex communication system.

\section{MMSE BEAMFORMING DESIGNS}

Precoding matrices must be designed to eliminate the selfinterference signal but still ensure the limited transmit power at each node ( $\operatorname{trace}\left(\mathbf{V}_{i} \mathbf{V}_{i}^{H}\right) \leq P, i=1,2$, where $P$ is the transmit power constrain).

From (3) and (4), desired signal MSE at node $i$ can be represented as

$$
\begin{aligned}
E_{i}= & \mathbf{T}_{i} \mathbf{G}_{j} \mathbf{V}_{j} \mathbf{V}_{j}^{\mathrm{H}} \mathbf{G}_{j}^{H} \mathbf{T}_{i}^{H}-\mathbf{T}_{i} \mathbf{G}_{j} \mathbf{V}_{j}-\mathbf{V}_{j}^{\mathrm{H}} \mathbf{G}_{j}^{H} \mathbf{T}_{i}^{H}+\mathbf{I} \\
& +\mathbf{T}_{i} \mathbf{H}_{i} \mathbf{V}_{i} \mathbf{V}_{i}^{\mathrm{H}} \mathbf{H}_{i}^{H} \mathbf{T}_{i}^{H}+\sigma^{2} \mathbf{T}_{i} \mathbf{T}_{i}^{H}, \\
& i=1,2, j=(i \bmod 2)+1 .
\end{aligned}
$$

The desired signal MSE minimization under the per-node power constraint can be formulated as

$$
\begin{gathered}
\min \sum_{i=1}^{2} \operatorname{trace}\left(E_{i}\right), \\
\text { subject to: trace }\left(\mathbf{V}_{i} \mathbf{V}_{i}^{H}\right) \leq P .
\end{gathered}
$$

To solve (7), we construct the Lagrangian function as

$\mathcal{L}\left(\mathbf{T}_{i}, \mathbf{V}_{i}, \lambda_{i}\right)=\mathbf{T}_{1} \mathbf{G}_{2} \mathbf{V}_{2} \mathbf{V}_{2}^{\mathrm{H}} \mathbf{G}_{2}^{H} \mathbf{T}_{1}^{H}-\mathbf{T}_{1} \mathbf{G}_{2} \mathbf{V}_{2}-\mathbf{V}_{2}^{\mathrm{H}} \mathbf{G}_{2}^{H} \mathbf{T}_{1}^{H}+$

$\mathbf{T}_{2} \mathbf{G}_{1} \mathbf{V}_{1} \mathbf{V}_{1}^{\mathrm{H}} \mathbf{G}_{1}^{H} \mathbf{T}_{2}^{H}-\mathbf{T}_{2} \mathbf{G}_{1} \mathbf{V}_{1}-\mathbf{V}_{1}^{\mathrm{H}} \mathbf{G}_{\mathbf{1}}^{H} \mathbf{T}_{2}^{H}+2 \mathbf{I}+$

$\lambda_{1}\left(\operatorname{trace}\left(\mathbf{V}_{1} \mathbf{V}_{1}^{H}\right)-P\right)+\lambda_{2}\left(\operatorname{trace}\left(\mathbf{V}_{2} \mathbf{V}_{2}^{H}\right)-P\right)$,
The Karush-Kuhn-Tucker (KKT) conditions [12] for (8) for $i=1,2$ are given by

$$
\begin{gathered}
\frac{\partial \mathcal{L}\left(\mathbf{T}_{i}, \mathbf{V}_{i}, \lambda_{i}\right)}{\partial \mathbf{T}_{i}^{*}}=\mathbf{T}_{i} \mathbf{G}_{j} \mathbf{V}_{j} \mathbf{V}_{j}^{\mathrm{H}} \mathbf{G}_{j}^{H}-\mathbf{V}_{j}^{\mathrm{H}} \mathbf{G}_{j}^{H} \\
\quad+\mathbf{T}_{i} \mathbf{H}_{i} \mathbf{V}_{i} \mathbf{V}_{i}^{\mathrm{H}} \mathbf{H}_{i}^{H}+\sigma^{2} \mathbf{T}_{i}=0 \\
\frac{\partial \mathcal{L}\left(\mathbf{T}_{i}, \boldsymbol{V}_{i}, \lambda_{i}\right)}{\partial V_{i}^{*}}=\mathbf{G}_{i} \mathbf{T}_{j} \mathbf{T}_{j}^{\mathrm{H}} \mathbf{G}_{i}^{H} \mathbf{V}_{i}^{H}-\mathbf{G}_{i} \mathbf{T}_{j}^{H} \\
\quad+\mathbf{H}_{i}^{\mathrm{H}} \mathbf{T}_{i}^{H} \mathbf{T}_{i} \mathbf{H}_{i} \mathbf{V}_{i}+\lambda_{i} \mathbf{V}_{i}=0 \\
\frac{\partial \mathcal{L}\left(\boldsymbol{T}_{i}, \boldsymbol{V}_{i}, \lambda_{i}\right)}{\partial \lambda_{i}}=\operatorname{trace}\left(\mathbf{V}_{i} \mathbf{V}_{i}^{H}\right)-P=0
\end{gathered}
$$

Finally, the optimal pre/postcoding matrix are computed as

$$
\begin{aligned}
\mathbf{T}_{i} & =\mathbf{V}_{j}^{\mathrm{H}} \mathbf{G}_{j}^{H}\left(\mathbf{G}_{j} \mathbf{V}_{j} \mathbf{V}_{j}^{\mathrm{H}} \mathbf{G}_{j}^{H}+\mathbf{H}_{i} \mathbf{V}_{i} \mathbf{V}_{i}^{\mathrm{H}} \mathbf{H}_{i}^{H}+\sigma^{2} \mathbf{I}\right)^{-1}, \\
\mathbf{V}_{i} & =\left(\mathbf{G}_{i}^{H} \mathbf{V}_{j}^{H} \mathbf{G}_{j} \mathbf{G}_{j}+\mathbf{H}_{i} \mathbf{V}_{i} \mathbf{V}_{i}^{\mathrm{H}} \mathbf{H}_{i}^{H}+\lambda_{i} \mathbf{I}\right)^{-1} \mathbf{G}_{i} \mathbf{T}_{j},
\end{aligned}
$$

where $\lambda_{i}$ satisfying the individual power constraint in (11) (with $\mathbf{V}_{i}$ is given) can be found by bisection root method.

The overall proposed MSE minimization scheme is summarized below:

ALGORITHM 1. MSE-BASED BEAMFORMING DESIGN FOR MIMO FULL-DUPLEX COMMUNICATION SYSTEM

\section{Initialization: \\ - Choose an initial value $\mathbf{V}_{i}$ satisfying the power constraint in (11), \\ While (not converge) \\ - For fixed $\mathbf{V}_{i}$, calculate $\mathbf{T}_{i}$ according to (12). \\ - For found $\mathbf{T}_{i}$, calculate $\lambda_{i}$ according to (11). \\ - Update $\mathbf{V}_{i}$ according to (13). \\ end}

\section{SiMULATION RESULTS}

In this section, computer simulations are conducted to provide numerical results for illustrating the performance of proposed beamforming algorithms. In the figures of numerical results, each plotted point of the system performance is obtained by averaging over 1000 independent channel realizations.

In the simulated system, signal power is normalized and the system SNR is defined as $\frac{1}{\sigma^{2}}$. In generating channel responses, elements of $\mathbf{H}_{i}, \mathbf{G}_{i}$ are assumed to be independently random complex values following $\aleph(0,1)$. The channel response is assumed to be a block-fading. Modulation types include BPSK, QPSK, 16-QAM and 64-QAM.

TABLE1

THE SELF-INTERFERENCE CHANNEL MATRIX IN NODE 1 (H1)

\begin{tabular}{llll}
\hline$-1.141-0.2877 \mathrm{i}$ & $0.3114+0.7959 \mathrm{i}$ & $-0.2050+0.1065 \mathrm{i}$ & $0.8151+0.9146 \mathrm{i}$ \\
\hline$-0.5564-0.2107 \mathrm{i}$ & $1.0281+0.9919 \mathrm{i}$ & $0.9278+0.0815 \mathrm{i}$ & $0.8910+0.8768 \mathrm{i}$ \\
\hline
\end{tabular}

TABLE 2

THE PRECODING MATRIX IN NODE 1 (V1)

\begin{tabular}{ll}
\hline $0.7069+0.0000 \mathrm{i}$ & $-0.0004-0.0004 \mathrm{i}$ \\
\hline$-0.0004+0.0004 \mathrm{i}$ & $0.7074-0.0000 \mathrm{i}$ \\
\hline
\end{tabular}


TABLE 3

THE POSTCODING MATRIX IN NODE 1 (T1)

\begin{tabular}{cc}
\hline $0.0344-0.5331 \mathrm{i}$ & $0.5592+0.0158 \mathrm{i}$ \\
\hline$-0.2918-0.5749 \mathrm{i}$ & $-0.6127-0.0062 \mathrm{i}$ \\
\hline $0.0200+0.6798 \mathrm{i}$ & $0.0410+0.1043 \mathrm{i}$ \\
\hline$-0.0823+0.0330 \mathrm{i}$ & $0.8737-0.1396 \mathrm{i}$ \\
\hline
\end{tabular}

TABLE 4

THE DESIRED-SIGNAL CHANNEL MATRIX IN NODE $1(G 2)$

\begin{tabular}{llll}
\hline$-0.1713+1.2413 \mathrm{i}$ & $-0.5679+1.3452 \mathrm{i}$ & $-0.0835+0.2912 \mathrm{i}$ & $-0.2542+0.1041 \mathrm{i}$ \\
\hline $0.3325+0.0235 \mathrm{i}$ & $-0.3507-0.8347 \mathrm{i}$ & $-0.4733-0.6575 \mathrm{i}$ & $1.1609-0.3360 \mathrm{i}$ \\
\hline
\end{tabular}

TABLE 5

THE PRECODING MATRIX IN NODE 2 (V2)

\begin{tabular}{ll}
\hline $0.7070-0.0000 \mathrm{i}$ & $0.0001-0.0001 \mathrm{i}$ \\
\hline $0.0001+0.0001 \mathrm{i}$ & $0.7072+0.0000 \mathrm{i}$
\end{tabular}

TABLE 6

THE SELF-INTERFERENCE BEAMFORMING MATRICES IN NODE $1\left(\boldsymbol{T}_{1} \boldsymbol{H}_{1} \boldsymbol{V}_{1}\right)$

\begin{tabular}{ll}
\hline $0.0002+0.0011 \mathrm{i}$ & $0.0007-0.0001 \mathrm{i}$ \\
\hline$-0.0003-0.0011 \mathrm{i}$ & $-0.0008+0.0008 \mathrm{i}$ \\
\hline
\end{tabular}

Tables 1-7 are matrices in the point-to-point full-duplex communication system with the antenna configuration $\left(N_{R}=\right.$ 2, $N_{T}=4$ ). In this tables, the channel matrices are randomly generated in loops. From these matrices, we find the beamforming matrices in formulas (12) and (13), then check whether constraints (7). Tables 1,2 and 3 represent the matrices at node 1, including self-interference channel matrix $\mathbf{H}_{1}$, the precoding matrix $\mathbf{V}_{l}$ and the post coding matrix $\mathbf{T}_{1}$. Tables 4 and 5 represent the matrices at node 2, including the desired signal channel matrix $\mathbf{G}_{2}$ and the precoding matrix $\mathbf{V}_{2}$. In Table 6, we introduce the self-interference beamforming matrices in node $1\left(\mathbf{T}_{1} \mathbf{H}_{1} \mathbf{V}_{1}\right)$. We can see that the selfinterfering signal is almost eliminated. In addition, in Table 7 , we introduce the desired signal beamforming matrices in node $1\left(\mathbf{T}_{1} \mathbf{G}_{2} \mathbf{V}_{2}\right)$. The result show that $\mathbf{T}_{1} \mathbf{G}_{2} \mathbf{V}_{2} \sim \mathbf{I}$, so the desired signal completely restored.

\section{TABLE 7}

THE DESIRED-SIGNAL BEAMFORMING MATRICES IN NODE $1\left(\boldsymbol{T}_{1} \boldsymbol{G}_{2} \boldsymbol{V}_{2}\right)$

\begin{tabular}{ll}
\hline $0.9988-.0000 \mathrm{i}$ & $-.0002+0.0001 \mathrm{i}$ \\
\hline$-0.0002-0.0001 \mathrm{i}$ & $0.9985+0.0000 \mathrm{i}$ \\
\hline
\end{tabular}

Fig. 2 shows the average sum-rate of the two systems with the antenna configuration $\left(N_{R}=4, N_{T}=8\right)$ and $\left(N_{R}=\right.$ $2, N_{T}=4$ ), respectively. The result show that the higher antenna configuration provides the greater signal speed.

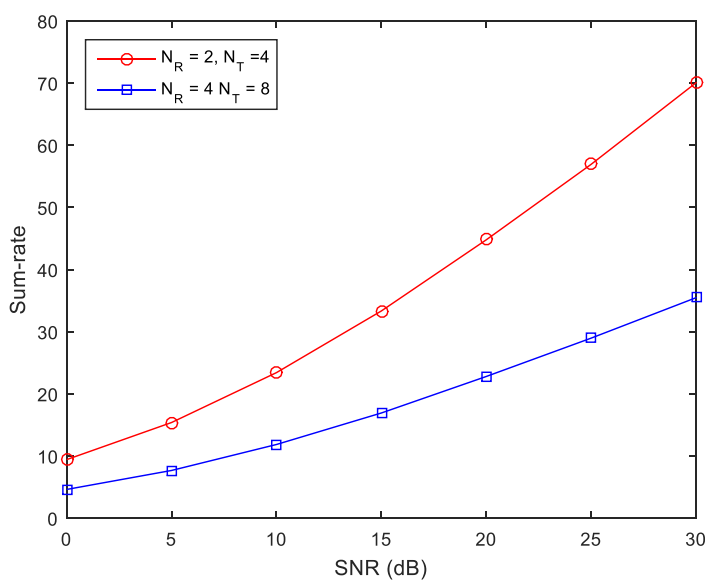

Fig. 2 Full-duplex beamforming Sum-rate versus SNR with difference antenna configurations

Fig. 3 shows the MSE vs SNR of the two systems with the antenna configuration $\left(N_{R}=4, N_{T}=8\right)$ and $\left(N_{R}=\right.$ $2, N_{T}=4$ ), respectively. The result show that the higher antenna configuration will give the better performance.

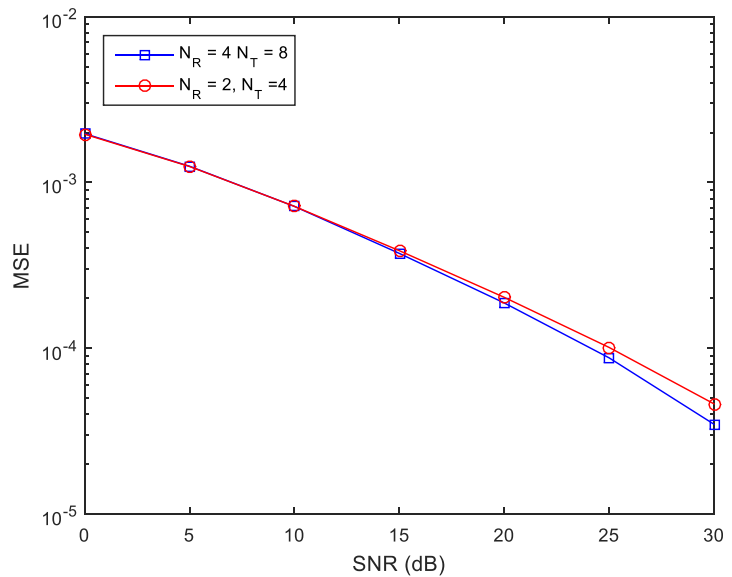

Fig. 3 Full-duplex beamforming MSE versus SNR with difference antenna configurations.

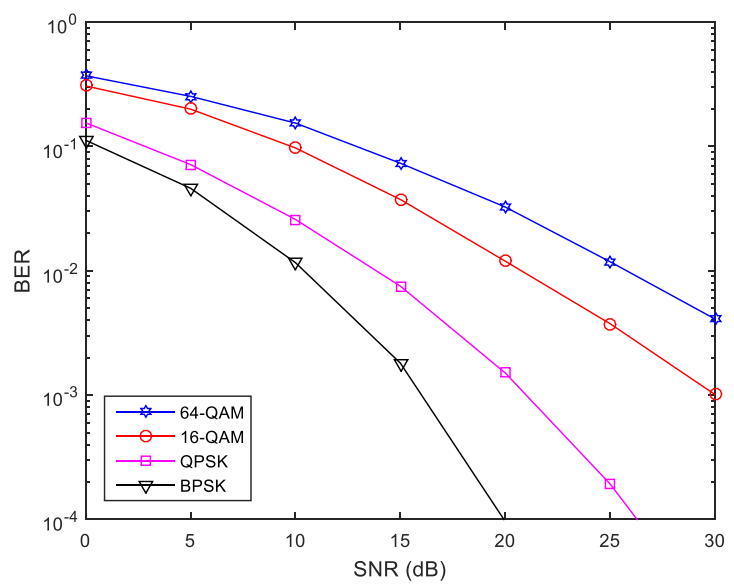

Fig. 4 Full-duplex beamforming BER versus SNR with the antenna configuration $\left(N_{R}=4, N_{T}=8\right)$. 
Fig. 4 shows bit error rate (BER) versus SNR in the with modulation types BPSK, QPSK, 16-QAM and 64-QAM, respectively. In this configuration, each node equipped $N_{R}=$ 4 receive antennas and $N_{T}=8$ transmit antennas. We can see that, BPSK gives the best results and 64-QAM gives the worst result. As many constellations are more susceptible to interference and interference.

Fig. 2, 3 and 4 show that the proposed algorithm fullduplex beamforming provides good performance because the self-interference almost eliminated completely.

\section{CONCLUSION}

This paper considered the problem of MIMO full-duplex bidirectional communication between a pair of MIMO modems in the presence of noise and self-interference. We proposed a beamforming scheme base on minimizing total mean square errors under the individual transmit power constraints. From the results obtained, it was found that the proposed algorithm full-duplex beamforming provides good performance because the self-interference almost eliminated completely.

\section{NOMENCLATURE}

$\begin{array}{ll}\text { BER } & \text { Bit Error Rate } \\ \text { BPSK } & \text { Binary phase-shift keying } \\ \text { DS } & \text { Desire Signal } \\ \text { FD } & \text { Full-Duplex } \\ \text { MIMO } & \text { Multiple input/multiple output } \\ \text { MMSE } & \text { Minimum mean square error } \\ \text { QAM } & \text { Quadrature amplitude modulation } \\ \text { QPSK } & \text { Quadrature phase-shift keying } \\ \text { SI } & \text { Self-Interference }\end{array}$

\section{REFERENCES}

[1] D. Kim, H. Lee and D. Hong, "A Survey of In-Band Full-Duplex Transmission: From the Perspective of PHY and MAC Layers," IEEE Commun. Surveys\& Tutorials, Vol. 17, No. 4, pp. 2017 - 2046, Nov. 2015.

[2] A. Masmoudi, Tho Le-Ngoc, "Residual self-interference after cancellation in full-duplex systems," in Proc. IEEE International Conference, pp. 4680-4685, Jun. 2014.

[3] M. Duarte, A. Sabharwal, "Full-duplex wireless communications using off-the-shelf radios: feasibility and first results," in Proc. ASILOMAR Signals, Syst., Comput., pp. 1558-1562, Nov. 2010.

[4] G. J. Foschini and M. J. Gans. On limits of wireless communication in a fading environment when using multiple antennas. Wireless Personal Communications, pp. 311-335, March 1998.

[5] R. S. Blum, "MIMO capacity with interference," in Proc. Conf. Inform. Sci. Syst., Mar. 2002.

[6] B. G. Agee, "Exploitation of internode MIMO channel diversity in spatially distributed multipoint communication networks," in Proc. Asilomar Conf., Nov. 2001.

[7] Spencer, Quentin H., A. Lee Swindlehurst, and Martin Haardt. "Zeroforcing methods for downlink spatial multiplexing in multiuser MIMO channels." IEEE Transactions on Signal Processing 52.2, pp: 461-471, 2004.

[8] T. Riihonen, S. Werner, and R. Wichman, "Mitigation of loopback selfinterference in full-duplex MIMO relays," IEEE Trans. Signal Process., vol. 59, no. 12, pp. 5983-5993, Dec. 2011.

[9] B. Chun and H. Park, "A spatial-domain joint-nulling method of selfinterference in full-duplex relays," IEEE Commun. Lett., vol. 16, no. 4, pp. 436-438, Apr. 2012.

[10] H. Ju, E. Oh, and D. Hong, "Improving efficiency of resource usage in two-hop full duplex relay systems based on resource sharing and interference cancellation," IEEE Trans. Wireless Commun., vol. 8, no. 8, pp. 3933-3938, Aug. 2009.

[11] B. Day, A. Margetts, D. Bliss, and P. Schniter, "Full-duplex MIMO relaying: achievable rates under limited dynamic range," IEEE J. Sel. Areas Commun., vol. 30, no. 8, pp. 1541-1553, Sep. 2012.

[12] S. Boyd and L. Vandenberghe, Convex Optimization. Cambridge University Press, 2004.

[13] G. G. Raleigh and J. M. Cioffi, "Spatio-temporal coding for wireless communication," IEEE Trans. Commun., vol. 46, pp. 357-366, Mar. 1998 